\title{
Enhanced Morphological and Thermal Stabilities of Nickel Germanide with an Ultrathin Tantalum Layer Studied by Ex Situ and In Situ Transmission Electron Microscopy
}

\author{
Jae-Wook Lee, ${ }^{1}$ Hyung-Kyu Kim, ${ }^{1}$ Jee-Hwan Bae, ${ }^{1}$ Min-Ho Park, ${ }^{1}$ Hyoungsub Kim, ${ }^{1}$ \\ Jiho Ryu, ${ }^{2}$ and Cheol-Woong Yang ${ }^{1, \star}$ \\ ${ }^{1}$ School of Advanced Materials Science \& Engineering, Sungkyunkwan University, Suwon 440-746, South Korea \\ ${ }^{2}$ Department of Automobile Development, Ajou Motor College, Boryeong 355-769, South Korea
}

\begin{abstract}
The formation and morphological evolution of germanides formed in a ternary Ni/Ta-interlayer/Ge system were examined by ex situ and in situ annealing experiments. The Ni germanide film formed in the $\mathrm{Ni} / \mathrm{Ta}$-interlayer/Ge system maintained continuity up to $550^{\circ} \mathrm{C}$, whereas agglomeration of the Ni germanide occurred in the Ni/Ge system without Ta-interlayer. Through microstructural and chemical analysis of the $\mathrm{Ni} / \mathrm{Ta}$-interlayer/Ge system during and after in situ annealing in a transmission electron microscope, it was confirmed that the Ta atoms remained uniformly on the top of the newly formed Ni germanide layer during the diffusion reaction. Consequently, the agglomeration of the Ni germanide film was retarded and the thermal stability was improved by the Ta incorporation.
\end{abstract}

Key words: Ni germanide, thermal stability, in situ TEM, Ta-interlayer, agglomeration, AEM

\section{INTRODUCTION}

Because of the ever-increasing demand for scaling down metal-oxide-semiconductor field-effect transistor (MOSFET) devices, Si-based devices are approaching their fundamental limit. Recently, Ge has been considered as an attractive replacement for Si substrate in high-performance transistors because of its higher carrier mobility. Compared with $\mathrm{Si}$, Ge offers two times higher intrinsic electron mobility and four times higher intrinsic hole mobility (Martin et al., 1989; Lee et al., 2001; Shang et al., 2003; Saraswat et al., 2006), and these enhanced mobilities result in larger saturated drain currents, enhanced transconductance, and higher cutoff frequencies (Spann et al., 2005). However, there are several problems in realizing Ge-based MOSFETs, such as the inferior thermal stability and water solubility of Ge oxides. Successful studies on Ge MOSFETs with high- $k$ gate dielectric such as Ge oxynitride, $\mathrm{HfO}_{2}$, and $\mathrm{ZrO}_{2}$ have been demonstrated recently (Gusev et al., 2004; Bai et al., 2006; Oh et al., 2007). These very promising results suggest that the use of Ge channels will allow MOSFET technology to be extended beyond the limitations imposed by the material properties of Si.

In order to fully exploit the superior transport properties of Ge, a low-resistance contact technology will have to be developed based on metal germanides. A self-aligned germanide through the solid-phase reactions of $\mathrm{Ge}$ and metals, such as $\mathrm{Ti}, \mathrm{Co}$, and $\mathrm{Ni}$, in much the same way as self-aligned silicide, is known to be effective in reducing the parasitic source/drain resistance (Ashburn et al., 1992; Patterson et al., 1994; Park et al., 2009). Of these metal germanides, the Ni germanide is highly attractive as a promising

() MICROSCOPY SOCIETY OF AMERICA 2013

${ }^{\star}$ Corresponding author. E-mail: cwyang@skku.edu self-aligned germanide material for Ge MOSFET technology because of its low processing temperature and low resistivity (Hsu et al., 2005; Zhang et al., 2005). However, the poor thermal stability of $\mathrm{Ni}$ germanide due to agglomeration is one major drawback. Several methods to slightly improve the thermal stability of $\mathrm{Ni}$ germanide such as using $\mathrm{Ni}$ alloy targets and ultrathin $\mathrm{Ti}$ and $\mathrm{Yb}$ interlayers have been reported (Liew et al., 2006; Zhu et al., 2007; Zhang et al., 2009).

This study examined the formation and microstructural evolution of $\mathrm{Ni}$ germanide utilizing an ultrathin $\mathrm{Ta}$ interlayer as a function of annealing temperature using $e x$ situ and in situ annealing experiments. The in situ annealing experiments were carried out in a transmission electron microscope (TEM) equipped with a specimen heater. And various analytical electron microscopy (AEM) techniques were used to investigate the microstructure and chemical composition of the phase formed by the reaction.

\section{Materials and Methods}

First, $p$-type ( $\left.\begin{array}{lll}1 & 0 & 0\end{array}\right)$ Ge substrates were RCA cleaned and dipped cyclically into a dilute hydrofluoric acid (HF) solution ( $\left.\mathrm{HF}: \mathrm{H}_{2} \mathrm{O}=1: 100\right)$ and deionized water for $30 \mathrm{~s}$, respectively, five times, and were immediately loaded into a DC magnetron sputtering system. Then $\sim 2$-nm-thick Ta and 13-nm-thick Ni films were deposited sequentially at room temperature in a 5.0 mtorr using Ar gas after reaching a base pressure below $2.0 \times 10^{-7}$ torr. A cross-sectional highresolution (HR) TEM micrograph of the as-deposited specimen of the $\mathrm{Ni} / \mathrm{Ta}$-interlayer/Ge system formed by this way was shown in the inset of Figure 1. Approximately 15-nmthick Ni film alone was also deposited on Ge substrates for comparison. For the formation of Ni germanide, ex situ and 


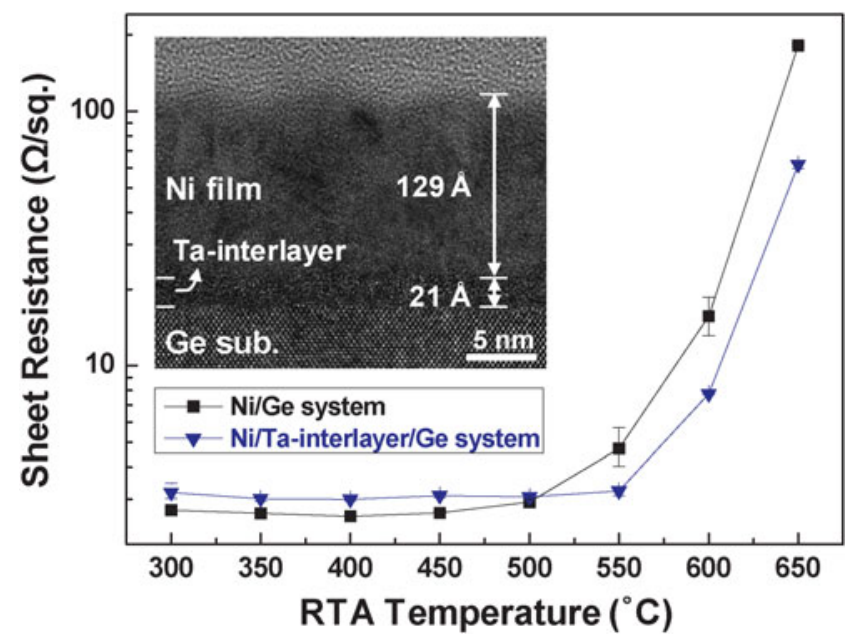

Figure 1. The $R_{\mathrm{s}}$ values of the $\mathrm{Ni} / \mathrm{Ge}$ and $\mathrm{Ni} / \mathrm{Ta}$-interlayer/Ge systems after rapid thermal annealing (RTA) in the temperature range between 300 and $650^{\circ} \mathrm{C}$ for $60 \mathrm{~s}$ in $\mathrm{N}_{2}$ ambient. Inset shows a cross-sectional high-resolution transmission electron microscope micrograph of the as-deposited specimen of the Ni/Ta-interlayer/Ge system formed by this experimental procedure.

in situ annealing experiments were carried out. For the ex situ annealing experiment, each sample was annealed by rapid thermal annealing (RTA) at every $50^{\circ} \mathrm{C}$ temperature interval in the temperature range from 300 to $650^{\circ} \mathrm{C}$ for $60 \mathrm{~s}$ in $\mathrm{N}_{2}$ ambient. The unreacted metal layer was selectively removed from a diluted phosphoric acid $\left(\mathrm{H}_{3} \mathrm{PO}_{4}\right)$ solution. The sheet resistance $\left(R_{\mathrm{s}}\right)$ was measured by using a fourpoint probe system. The microstructures and chemical compositions of the specimens were characterized by AEM (JEM-2100F, JEOL Co. Ltd.) equipped with scanning transmission electron microscope (STEM)/energy-dispersive spectrometer (EDS). TEM specimens were prepared using a tripod polisher for mechanical grinding and Ar-ion milling, and the TEM specimens for the in situ annealing experiment were fixed on Mo oval grids in order to reduce the occurrence of thermal drift phenomena due to thermal expansion. The in situ annealing experiment was carried out in a TEM (JEM-3011, JEOL Co. Ltd.) with a specimen heating-tilting holder (EM-31050, JEOL Co. Ltd.) and a heater control unit (EM-SHU2, JEOL Co. Ltd.). The images were recorded by using a CCD camera (2k Gatan Ultrascan) and a digital video recording system with a $1 / 30 \mathrm{~s}$ time resolution (30 frames/s) connected to a TV rate camera.

\section{Results And Discussion}

In order to compare the thermal stability of $\mathrm{Ni}$ germanides formed from the $\mathrm{Ni} / \mathrm{Ge}$ and $\mathrm{Ni} / \mathrm{Ta}$-interlayer/Ge systems, the specimen were annealed by RTA at the temperature range between 300 and $650^{\circ} \mathrm{C}$ for $60 \mathrm{~s}$. Figure 1 shows the $R_{\mathrm{s}}$ values of the specimens with and without the Ta-interlayer as a function of annealing temperature. For all the specimens of both systems, the measured $R_{\mathrm{s}}$ values were almost constant ( $\sim 3 \Omega /$ sq.) up to $500^{\circ} \mathrm{C}$, above which the measured $R_{\mathrm{s}}$ values in the $\mathrm{Ni} / \mathrm{Ge}$ system increased abruptly. It is
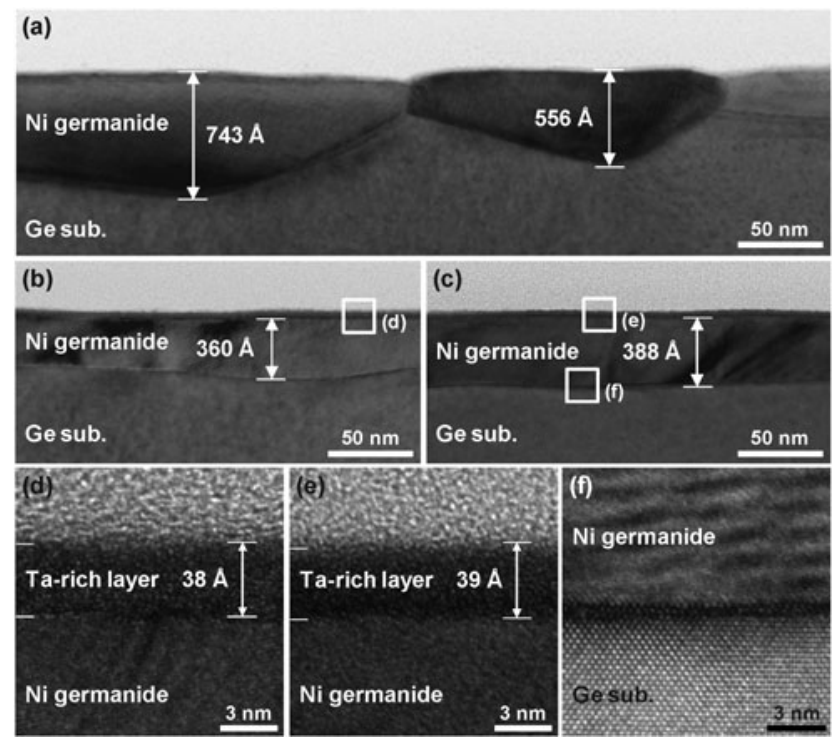

Figure 2. Cross-sectional transmission electron microscope (TEM) micrographs: (a) the Ni/Ge system annealed by rapid thermal annealing (RTA) at $550^{\circ} \mathrm{C}$ for $60 \mathrm{~s}$ in $\mathrm{N}_{2}$ ambient, and the $\mathrm{Ni} / \mathrm{Ta}$ interlayer/Ge system annealed by RTA at (b) $300^{\circ} \mathrm{C}$ and (c) $550^{\circ} \mathrm{C}$, and high-resolution TEM micrographs of two interfaces: Ta-rich layer/ $\mathrm{Ni}$ germanide in specimens annealed at (d) $300^{\circ} \mathrm{C}$ and (e) $550^{\circ} \mathrm{C}$, and (f) $\mathrm{Ni}$ germanide/Ge substrate.

noteworthy that the measured $R_{\mathrm{s}}$ value of the specimen annealed at $550^{\circ} \mathrm{C}$ in the $\mathrm{Ni} / \mathrm{Ta}$-interlayer/Ge system maintained almost constant value which was measured by specimens annealed at lower temperatures. In the case of $\mathrm{Ni}$ germanide, the degradation of the $R_{\mathrm{s}}$ value by hightemperature annealing can be explained in terms of the agglomeration of NiGe (Park et al., 2007). For the Ni/Tainterlayer/Ge system, the thermal stability of $\mathrm{Ni}$ germanide was improved, probably because of the retardation of the agglomeration process by the Ta atoms, which are discussed in detail in the following paragraphs.

Figure 2a shows a cross-sectional TEM micrograph of the $\mathrm{Ni} / \mathrm{Ge}$ system annealed by RTA at $550^{\circ} \mathrm{C}$, where the $R_{\mathrm{s}}$ value doubly increased the initial values. The $\mathrm{Ni}$ germanide grains grew to $\sim 55-75 \mathrm{~nm}$ in thickness, and became irregular with thermal grooves between the germanide grains on the surface and at the interface between the germanide and the Ge substrate. In addition, separations of the germanide layer due to agglomeration of germanide grains were observed in several places (not shown). In the case of the $\mathrm{Ni}$ /Ta-interlayer/Ge system, an $\sim 35$-nm-thick continuous and uniform $\mathrm{Ni}$ germanide layer was observed on the $\mathrm{Ge}$ substrate in the specimen annealed at $300^{\circ} \mathrm{C}$ (see Fig. 2b). And the growth of Ni germanide grains was suppressed and a continuous and uniformly thick Ni germanide layer $(<40$ $\mathrm{nm}$-thick) was observed in the specimen annealed at $550^{\circ} \mathrm{C}$ (see Fig. 2c). It is notable that the existence of the dark layer was observed on top of the germanide layer in the $\mathrm{Ni} / \mathrm{Ta}-$ interlayer/Ge system. Figures $2 \mathrm{~d}-2 \mathrm{f}$ show the enlarged HRTEM images of the two interfaces, i.e., the ambient/NiGe and the NiGe/Ge substrate, which were indicated by white 

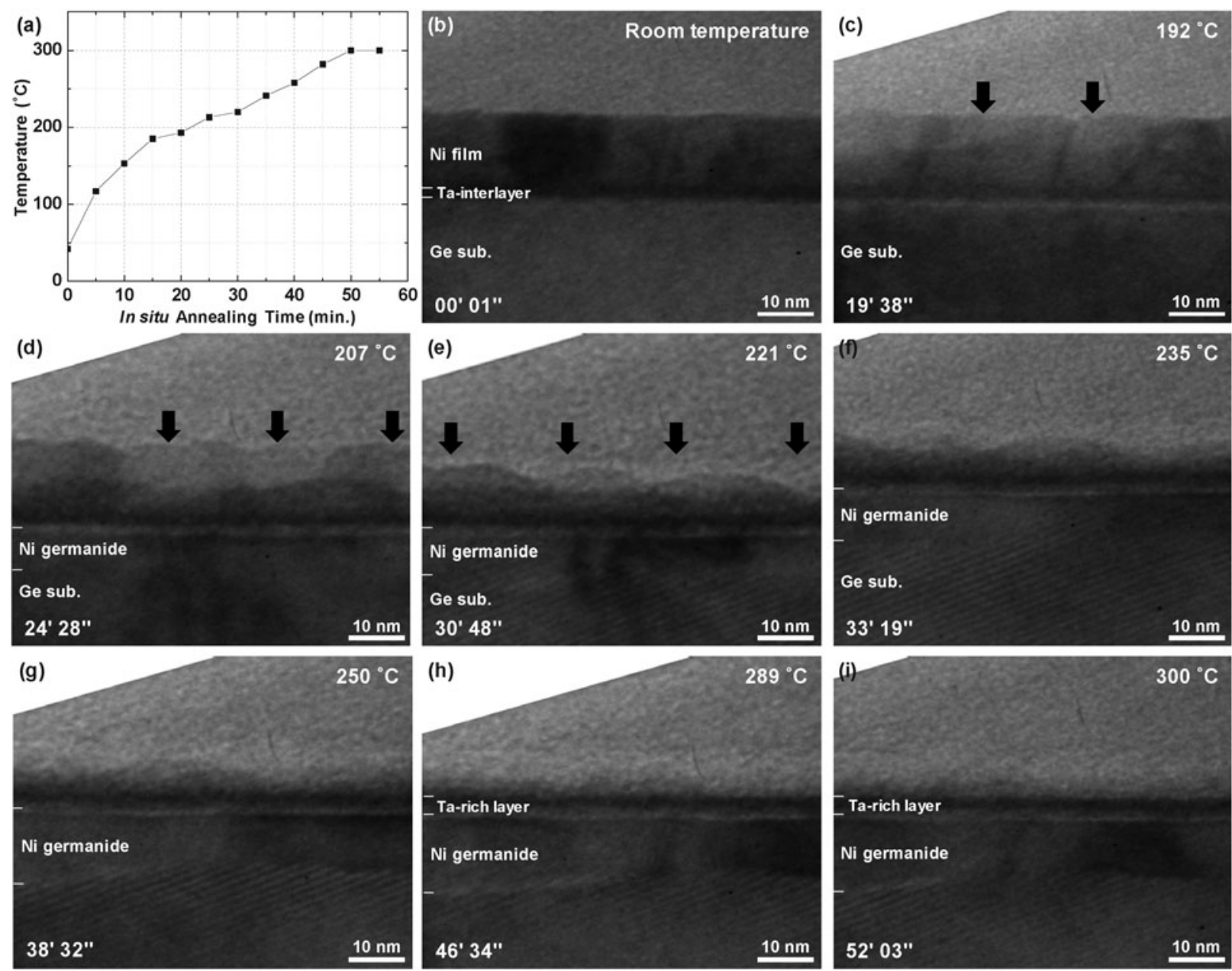

Figure 3. a: The temperature profile of the in situ annealing experiment. b-i: Individual frames selected from video sequence recorded during the in situ annealing of a cross-section of the $\mathrm{Ni} / \mathrm{Ta}$-interlayer/Ge system at temperature ranging from room temperature to $300^{\circ} \mathrm{C}$.

squares in Figures $2 \mathrm{~b}$ and $2 \mathrm{c}$. Approximately $4-\mathrm{nm}$-thick amorphous layers were formed on the NiGe films, as shown in Figures $2 \mathrm{~d}$ and 2e. These additional layers were confirmed to be a Ta-rich layer by EDS analysis. The compositions of the Ta-rich layers of the specimens annealed at 300 and $550^{\circ} \mathrm{C}$ were similar to each other and $44.60 \pm 1.26$ at.\% $\mathrm{Ta}, 21.56 \pm 2.13$ at. $\% \mathrm{Ni}, 33.84 \pm 2.62$ at. $\% \mathrm{Ge}$, and $44.35 \pm$ 1.30 at. $\% \mathrm{Ta}, 21.37 \pm 2.23$ at. $\% \mathrm{Ni}, 34.28 \pm 2.72$ at.\% Ge, respectively. And the interface between NiGe and the Ge substrate was clean, without any interfacial layer (see Fig. 2f). The agglomeration, which is a major cause of the increased $R_{\mathrm{s}}$ value of $\mathrm{Ni}$ germanide, is derived from the driving force of the decreased interfacial energy or surface energy. It is believed that the Ta-rich layer acts to reduce the surface free energy of NiGe film with outside ambient. Thus, the agglomeration is retarded because of the formation of the Ta-rich layer on the top of the Ni germanide layer.

In order to obtain a better understanding of the formation and microstructural evolution of the germanide and Ta-rich top layer in the Ni/Ta-interlayer/Ge system, a direct observation of the reactions were carried out using in situ annealing TEM. Figure 3a shows the temperature profile of the in situ annealing experiment, and the heating rate was $\sim 5.16^{\circ} \mathrm{C} / \mathrm{min}$. Figures $3 \mathrm{~b}-3 \mathrm{i}$ show a series of individual frames selected from a video sequence recorded during the in situ annealing of a cross-section of the $\mathrm{Ni} / \mathrm{Ta}$-interlayer/Ge system at temperature ranging from room temperature to $300^{\circ} \mathrm{C}$. The as-deposited specimen of $\mathrm{Ni} / \mathrm{Ta}$-interlayer/Ge system at room temperature was revealed in Figure 3b, and this is essentially the same as the specimen used for ex situ annealing experiments. As the annealing temperature was increased to $193^{\circ} \mathrm{C}$ (see Fig. 3c), the upper parts of the $\mathrm{Ni}$ film indicated by the arrows began to vacate. When the annealing temperature was increased to $207^{\circ} \mathrm{C}$, the vacated parts near the surface of the film indicated by the arrows became larger (see Fig. 3d), and $\mathrm{Ni}$ germanide grains began to form by diffusion of the $\mathrm{Ni}$ atoms into Ge substrate through the Ta-interlayer. As the annealing temperature increased, the remaining film thickness decreased gradually and the Ni germanide grain grew thicker, as shown in Figures $3 \mathrm{e}-3 \mathrm{~h}$. When the annealing temperature was increased up to $300^{\circ} \mathrm{C}$, an $\sim 15$-nm-thick Ni germanide layer was formed and an $\sim 3$-nm-thick film remained on top of the newly formed Ni germanide layer (see Fig. 3i). 


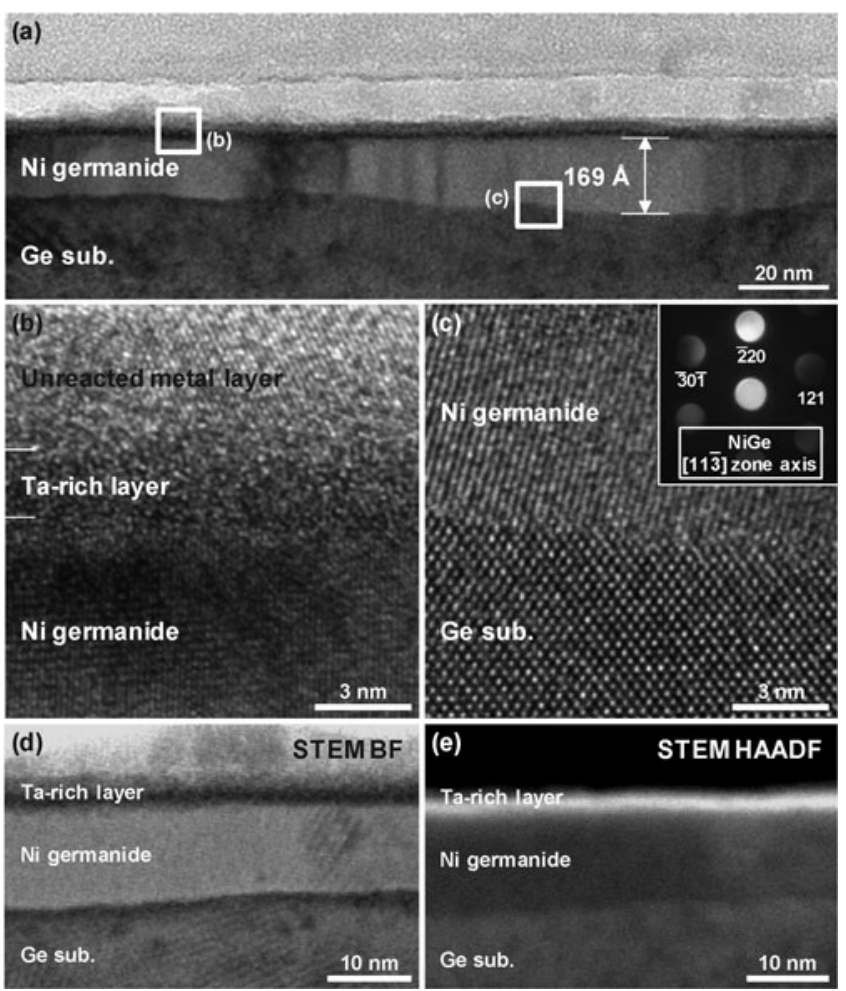

Figure 4. a: Low-magnification transmission electron microscope (TEM) micrograph of the in situ annealed specimen after annealing up to $300^{\circ} \mathrm{C}$, and high-resolution TEM micrographs of two interfaces. b: Ta-rich layer/Ni germanide and (c) Ni germanide/Ge substrate and nano-beam electron diffraction pattern obtained from a Ni germanide grain (inset), and scanning transmission electron microscope (d) bright field and (e) high-angle annular dark-field micrographs of the in situ annealed specimen.

For the characterization of the phases formed during the in situ annealing experiment, the microstructure and chemical composition were examined by using AEM equipped with STEM/EDS. Figure 4 shows the TEM micrographs of the specimen annealed for up to $300^{\circ} \mathrm{C}$ along with nano-beam electron diffraction (NBED) pattern obtained from the Ni germanide grain. The surface morphology of the Ta-rich layer and Ni germanide was relatively uniform; however, the interface between the $\mathrm{Ni}$ germanide and Ge substrate was slightly rougher than one of the ex situ annealed specimens (see Fig. 4a). Nevertheless, as shown in the HR-TEM micrographs of Figures $4 \mathrm{~b}$ and $4 \mathrm{c}$, the Ta-rich layer and the phase of $\mathrm{Ni}$ germanide formed from in situ annealing process were similar to one of ex situ annealed specimens. The average composition of the Ta-rich layer was $44.45 \pm 1.73$ at. $\%$ Ta, $21.16 \pm 2.97$ at. $\% \mathrm{Ni}$, and $34.38 \pm$ 3.60 at.\% Ge as measured by STEM/EDS utilizing a $0.7 \mathrm{~nm}$ electron probe. As shown in the STEM bright field (see Fig. 4d) and high-angle annular dark-field (HAADF, see Fig. 4e) micrographs, the contrast of the Ta-rich layer was distinct from the other phases. Considering the contrast mechanisms of STEM imaging, this difference in contrast was attributed to a mass-thickness effect on STEM imaging. $\mathrm{Ta}$ is the heaviest element among the components and is brighter in the STEM/HAADF image. And the NBED pattern (see the inset of Fig. 4c) obtained from a $\mathrm{Ni}$ germanide grain was indexed as the $[11 \overline{3}]$ zone axis pattern of NiGe, which has an orthorhombic structure: $a=5.811$, $b=5.381$, and $c=3.428 \AA$. This is consistent with the STEM/EDS result of $50.35 \pm 2.01$ at. $\% \mathrm{Ni}$ and $49.65 \pm 1.63$ at.\% Ge, which is close to the stoichiometric NiGe composition. Therefore, the phase of $\mathrm{Ni}$ germanide formed using this in situ annealing experiment was confirmed to be a $\mathrm{Ni}$ mono-germanide (NiGe). No other form of germanide was observed in this study.

The formation process of the $\mathrm{Ni}$ germanide and the Ta-rich layer in the Ni/Ta-interlayer/Ge system was analogous to one in the Ni-Ta alloy/Ge system of our previous work (Lee et al., 2008). Several intermetallic compounds can be present in the $\mathrm{Ta}-\mathrm{Ge}$ system. However, they cannot be formed under this annealing condition. In addition, the solid solubility of Ta in Ge is extremely low even at high temperature. Consequently, most of Ta atoms cannot diffuse into the Ge substrate and remain on top of the newly formed NiGe film. The presence of a Ta-rich layer on top of the Ni germanide layer likely modified the interfacial energies and suppressed the agglomeration of NiGe grains, thus improving the surface morphology of the $\mathrm{Ni}$ germanide formed in the $\mathrm{Ni} / \mathrm{Ta}$-interlayer/Ge system. As a result, the thermal stability of the $\mathrm{Ni} / \mathrm{Ta}$-interlayer/Ge system was better than that of the $\mathrm{Ni} / \mathrm{Ge}$ system.

\section{CONCLUSIONS}

This study examined the formation and morphological evolution of germanides formed in $\mathrm{Ni} / \mathrm{Ge}$ and $\mathrm{Ni} / \mathrm{Ta}-$ interlayer/Ge systems as a function of the annealing temperature using ex situ and in situ annealing experiments. The $\mathrm{Ni} / \mathrm{Ta}$-interlayer/Ge system improved the thermal stability and maintained low $R_{\mathrm{s}}$ value up to $550^{\circ} \mathrm{C}$. For the $\mathrm{Ni} / \mathrm{Ge}$ system annealed by RTA, severe agglomeration began to occur at an annealing temperature of $550^{\circ} \mathrm{C}$. On the other hand, in the case of the $\mathrm{Ni} / \mathrm{Ta}$-interlayer/Ge system, less agglomeration and a uniform surface morphology were observed under the same annealing condition. It is confirmed that the Ta-rich layer formed because Ta atoms remained on top of the newly formed $\mathrm{Ni}$ germanide film through AEM analysis of the $\mathrm{Ni} / \mathrm{Ta}$-interlayer/Ge system during and after the in situ annealing experiments. Eventually, the surface free energy of the Ni germanide film was reduced by this Ta-rich layer, and the thermal stability of the Ni germanide was improved.

\section{ACKNOWLEDGMENTS}

This work was supported by the National Research Foundation of Korea (NRF) grants funded by the Korea government (MEST, No. 2011-0017257, No. 2011-0019984, and No. 2011-0030803). The authors gratefully appreciate technical support from the cooperative center for research facilities (CCRF) at Sungkyunkwan University. 


\section{RefERENCES}

Ashburn, S.P., Öztürk, M.C., Wortman, J.J., Harris, G., HoneyCutT, J. \& MAher, D.M. (1992). Formation of titanium and cobalt germanides on $\mathrm{Si}(100)$ using rapid thermal processing. $J$ Electron Mater 21, 81-86.

Bai, W.P., Lu, N., Ritenour, A., Lee, M.L., Antoniadis, D.A. \& Kwong, D.L. (2006). Ge n-MOSFETs on lightly doped substrates with high- $k$ dielectric and TaN gate. IEEE Electron Device Lett 27, 175-178.

Gusev, E.P., Shang, H., Copel, M., Gribelyuk, M., D’emic, C., Kozlowski, P. \& Zabel, T. (2004). Microstructure and thermal stability of $\mathrm{HfO}_{2}$ gate dielectric deposited on $\mathrm{Ge}(100)$. Appl Phys Lett 85, 2334-2336.

Hsu, S.L., Chien, C.H., Yang, M.J., Huang, R.H., Leu, C.C., Shen, S.W. \& YANG, T.H. (2005). Study of thermal stability of nickel monogermanide on single- and polycrystalline germanium substrates. Appl Phys Lett 86, 251906.

Lee, J.W., Bae, J.H., Park, M.H., Kang, H.B., Kim, H. \& Yang, C.W. (2008). Microstructural evolution of nickel-germanide in the $\mathrm{Ni}_{1-x} \mathrm{Ta}_{x} / \mathrm{Ge}$ systems during in situ annealing. J Vac Sci Technol A 26, 688-691.

Lee, M.L., Leitz, C.W., Cheng, Z., Pitera, A.J., Currie, M.T., Taraschi, G., Fitzgerald, E.A. \& Antoniadis, D.A. (2001). Strained Ge channel $p$-type metal-oxide-semiconductor fieldeffect transistors grown on $\mathrm{Si}_{1-x} \mathrm{Ge}_{x} / \mathrm{Si}$ virtual substrates. Appl Phys Lett 79, 3344-3346.

Liew, S.L., Lee, R.T.P., Lee, K.Y., Balakrisnan, B., Chow, S.Y., LAI, M.Y. \& CHI, D.Z. (2006). Enhanced morphological stability of NiGe films formed using $\mathrm{Ni}(\mathrm{Zr})$ alloy. Thin Solid Films 504, 104-107.

Martin, S.C., Hitt, L.M. \& Rosenberg, J.J. (1989). P-channel germanium MOSFET's with high channel mobility. IEEE Electron Device Lett 10, 325-326.

Oh, J., Majhi, P., Kang, C.Y., Yang, J.W., Tseng, H.H. \& Jammy, R. (2007). Thermal stability of nanoscale Ge metal-oxide- semiconductor capacitors with $\mathrm{ZrO}_{2}$ high- $k$ gate dielectrics on Ge epitaxial layers. Appl Phys Lett 90, 202102.

Park, K., An, C.H., Lee, M.S., Yang, C.W., Lee, H.J. \& Kim, H. (2009). Microstructural evolution and electrical characteristics of Co-germanide contacts on Ge. J Electrochem Soc 156, $\mathrm{H} 229-\mathrm{H} 232$.

Park, K., Lee, B.H., Lee, D., Ko, D.H., KwaK, K.H., Yang, C.W. \& KIM, H. (2007). A study on the thermal stabilities of the NiGe and $\mathrm{Ni}_{1-x} \mathrm{Ta}_{x} \mathrm{Ge}$ systems. J Electrochem Soc 154, H557-H560.

Patterson, J.K., Park, B.J., Ritley, K., Xiao, H.Z., Allen, L.H. \& Rockett, A. (1994). Kinetics of Ni/a-Ge bilayer reactions. Thin Solid Films 253, 456-461.

Saraswat, K., Chui, C.O., Krishnamohan, T., Kim, D., Nayfeh, A. \& Pethe, A. (2006). High performance germanium MOSFETs. Mat Sci Eng B 135, 242-249.

Shang, H., Oкоrn-Schimdt, H., Отt, J., Kozlowski, P., Steen, S., Jones, E.C., Wong, H.S.P. \& Hanesch, W. (2003). Electrical characterization of germanium p-channel MOSFETs. IEEE Electron Device Lett 24, 242-244.

Spann, J.Y., Anderson, R.A., Thornton, T.J., Harris, G., ThoMAS, S.G. \& TrACY, C. (2005). Characterization of nickel germanide thin films for use as contacts to $\mathrm{p}$-channel germanium MOSFETs. IEEE Electron Device Lett 26, 151-153.

Zhang, Q., Wu, N., Osipowicz, T., Bera, L.K. \& Zhu, C. (2005). Formation and thermal stability of nickel germanide on germanium substrate. Jpn J Appl Phys 44, L1389-L1391.

Zhang, Y.Y., OH, J., LI, S.G., Jung, S.Y., PARK, K.Y., Shin, H.S., Lee, G.W., Wang, J.S., Majhi, P., Tseng, H.H., Jammy, R., Bae, T.S. \& LEE, H.D. (2009). Ni germanide utilizing ytterbium interlayer for high-performance Ge MOSFETs. Electrochem SolidState Lett 12, H18-H20.

Zhu, S., Yu, M.B., Lo, G.Q. \& Kwong, D.L. (2007). Enhanced thermal stability of nickel germanide on thin epitaxial germanium by adding an ultrathin titanium layer. Appl Phys Lett 91, 051905 . 